\title{
$J^{3}$ LETTERS
}

\section{Fabrication of implantable microshunt using a novel channel sealing technique}

\begin{abstract}
Alioune Diouf, Gregory Reimann, and Thomas Bifano Boston University Photonics Center \& Department of Manufacturing Engineering, Boston, Massachusetts 02146 E-mail: aliouned@gmail.com
\end{abstract}

\begin{abstract}
This letter reports the development of a novel microshunt by combining biocompatible materials with wellestablished, robust microelectromechanical systems (MEMS) fabrication processes. The key features of our microshunt fabrication process are summarized as follows: (1) Control over the thickness of channels' enclosure, (2) channels' height scalable by multiple thick resist patterning, (3) structural material deposition by electroplating, and (4) an easy fabrication process suitable for mass production. These features were realized using electroplating for the gold structural material, photoresist sacrificial material, and e-beam evaporation of adhesion layers. The combination of these techniques allows precise control on the microchannels width, depth, and length. A novel channel sealing technique that is extendable to more complex geometries is also presented. The developed method involves using a thin gold seed layer deposited via evaporation prior to electroplating the last structural layer, the cover of the channels. This method enhanced the electroplating process, yielding more control of the final thickness of the cover layer compared to methods consisting of overgrowing the channels until they seal. () 2008 Society of Photo-Optical Instrumentation Engineers. [DOI: 10.1117/1.2955935]
\end{abstract}

Subject terms: microchannel; electroplating; microfluidic; gold; plating.

Paper 07085LR received Oct. 16, 2007; revised manuscript received Apr. 25, 2008; accepted for publication May 5, 2008; published online Jul. 11, 2008.

\section{Introduction}

As applications for microfluidic technologies grow, the commercialization and proliferation of miniaturized fluidic devices are currently limited by cost and the ability to scale to large volume production. In this letter, we report a microshunt applicable in glaucoma treatment for controlling and regulating intraocular pressure (IOP) of the human eye. ${ }^{1}$ The presented fabrication technique can be extended to many fluidic devices.

Microfluidic device manufacturers employ many MEMS fabrication methods to build embedded channels, such as wafer bonding, electroplating with thick resist, and sacrificial layer etching and deposition sealing. ${ }^{2-6}$ Using electroplating with thick resist is a low-cost, low-temperature process relatively simple and suitable for high volume production. ${ }^{7}$ It also extends material selection to other biologically benign materials, such as gold, which are easily electroplated. In previous fabrication processes that have

$1537-1646 / 2008 / \$ 25.00$ @ 2008 SPIE been reported using electroplating, enclosed microchannels are obtained by overgrowing the channel walls until closed. ${ }^{7}$ This is undesirable because it makes the devices thicker than intended, therefore, unsuitable for some applications. It is also impossible to pattern and build any subsequent layers on top of the microchannels. We present a new technique of sealing these channels and demonstrate the scalable and low-cost fabrication of an implantable microshunt.

\section{Design Considerations}

The gold microshunt architecture used in this study is shown in Fig. 1. This particular shunt is designed to meet overall size requirements, constrained by the available space in applicable organs (in-between the eye's anterior chamber and the distal or suprachoroidal end) and surgical implantation incisions. ${ }^{1}$

These $5 \mathrm{~mm} \times 3 \mathrm{~mm} \times 50 \mu \mathrm{m}$ gold shunts consist of three layers. The bottom and top layers are $10 \mu \mathrm{m}$ thick [Fig. 1(a)] and form the base and sealing for the microchannels. Holes are designed on the both ends to facilitate the displaced organs in meshing around the device and maintaining it in place. The larger hole is added for handling purposes during surgery. The middle layer [Fig. 1(b)] is primarily made of channel walls $50 \mu \mathrm{m}$ wide, $30-45 \mu \mathrm{m}$ tall, and $50 \mu \mathrm{m}$ apart. Depending on the need of the patient, a number of ingress holes are opened allowing fluid from the anterior chamber of the eye to enter the device, directed through the microchannels, and exit in the suprachoroidal region of the eye.

For flows at these scales $\left(D_{n} \sim 10^{-4} \mathrm{~m}\right.$ and smaller $)$, the Reynolds number (Re) is on the order of several hundred and the flow is assumed to be laminar. We use the pressuredrop equation (1) of flow (water) between parallel plates to obtain the geometrical design parameters: length $(L)$; width $(W)$, and height $(h)$. We also consider a steady, onedimensional flow and negligible external forces. With all these simplifications, and a laminar flow friction factor $f \operatorname{Re}=96,{ }^{8}$ the nondimensional pressure drop solution can be written as

$\Delta p=\rho \frac{96}{\operatorname{Re}} \frac{L}{D_{h}} \frac{\bar{V}^{2}}{2}$,

where $\rho$ is the density, $\bar{V}$ the average velocity of the fluid, and $D_{h}$ is the hydraulic diameter $\left[D_{h}=2 h W /(h+W)\right]$.

For effective flow in the normal eye maintains the IOP pressure at its physiological level of $10-20 \mathrm{~mm} \mathrm{Hg}$. We select a width of $50 \mu \mathrm{m}$ to maximize the number of channels in the $3 \mathrm{~mm}$ wide device, and a length of $3.3 \mathrm{~mm}$, with heights ranging from 30 to $45 \mu \mathrm{m} \quad(\operatorname{Re} \approx 23$ and $\Delta p$ $\approx 20 \mathrm{~mm} \mathrm{Hg}$ ).

\section{Experimental Details}

Figure 2 shows the microfabrication process of the device. The process uses two masks: the first mask is used for the base and cover layers of the shunt, and the second mask is used for molding the channel layer.

The fabrication process begins with cleaning the wafer using a piranha-etch bath and oxygen reactive ion etching (RIE). The wafer is then spin-coated with a thin resist (Shi- 


\section{$\mathrm{JM}^{3}$ LETTERS}

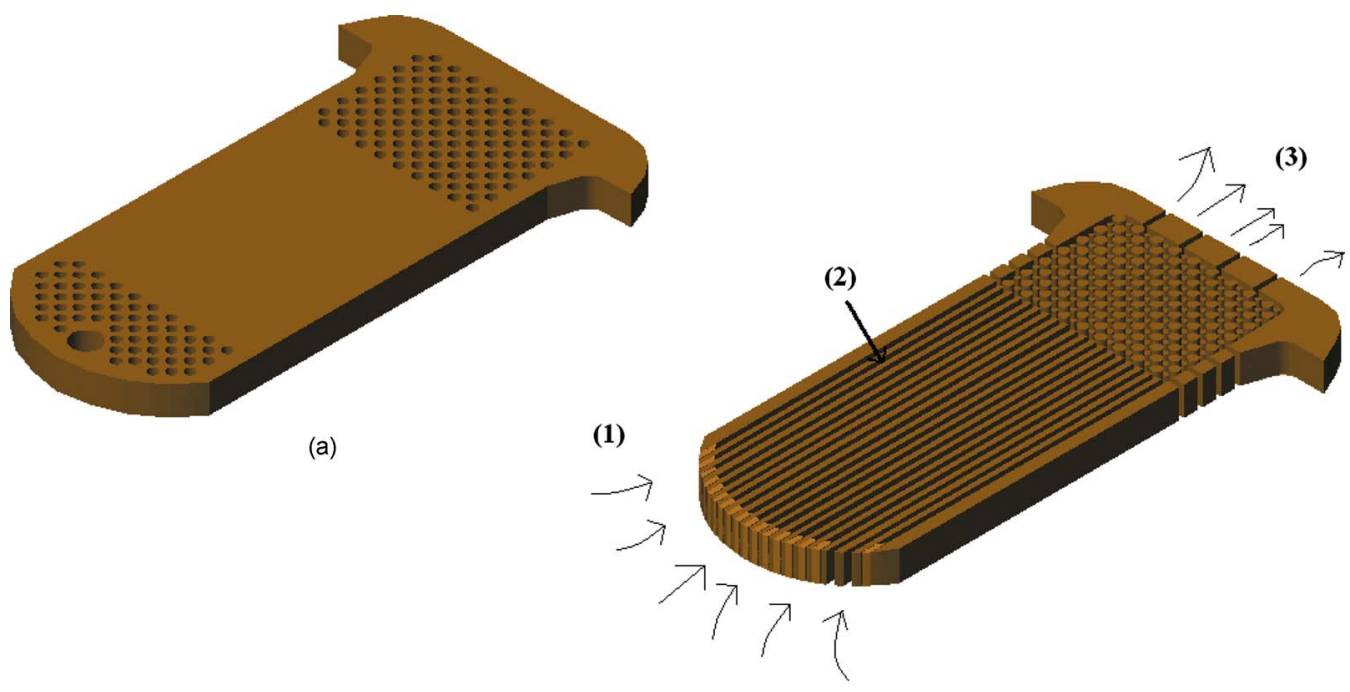

(b)

Fig. 1 (a) Bottom (layer 1) and top (layer 3) structures of the microshunt. (b) Microchannel layer (layer 2): (1) Fluid enters in ingress holes. Two holes shown opened; (2) fluid is directed through the internal microchannels; and (3) fluid exits the shunt into the suprachoroidal space.

pley, AZ 1813). A Cr/Au layer $(200 \AA / 1000 \AA)$ is then e-beam evaporated to form the adhesion (which will be removed once the device is completed) and seed layers, respectively. A portion of the gold seed layer also serves as a cathode for the electroplating process. The $\mathrm{Cr}$ and $\mathrm{Au}$ deposited onto the Si wafer are subsequently lifted off. The first layer [Fig. 2(a)] is then electroplated using a sodium sulfite-based gold electroplating solution, TechniGold 25E (Technic, Inc., Cranston, Rhode Island, USA) for the bath makeup. The electrochemical deposition was performed in a bath maintained at $40{ }^{\circ} \mathrm{C}$ and constantly agitated to promote even plating. The deposition was typically done with a current of $0.1-0.2 \mathrm{~mA}$ and deposition rate of $3.5-4.5 \mu \mathrm{m} / \mathrm{h}$ were observed with the $\mathrm{pH}$ between 6.5 and 6.8. Following the electroplating of the bottom layer, a $15 \mu \mathrm{m}$ positive photoresist is spun (SPR 220-7) and used as the mold for electroplating [Fig. 2(b)]. The $15 \mu \mathrm{m}$ thickness is achieved in a single coating and repeated twice or three times to reached the desired 30 or $45 \mu \mathrm{m}$ channel height. The removal of the edge bead resulting from this thick photoresist is necessary in order to get good contact during exposure. After developing, each thick resist lithography step is followed by RIE oxygen plasma cleaning to remove photoresist traces from the exposed gold surfaces (with negligible etching of the resist mold).

The fabrication of the resist mold is followed by the 30-45 $\mu \mathrm{m}$ thick electroplated gold layer [Fig. 2(c)]. As the device gets thicker, the processing of the resist becomes more stringent. The absorption coefficient of SPR220-7 (Shipley) is high, and at the desired thickness range $30-50 \mu \mathrm{m}$, either the bottom region is underexposed or the top region is overexposed. For this layer, the double- or triple-coat process is optimized to achieve straight and near-vertical sidewall profiles. After each coat, soft baking is carried out at a lower temperature and longer period than the preceding coat. This ensures that the all coats harden and the solvent evaporates in all layers of resist. Following this lithography step, the channel structures are electroplated using the aforementioned plating process.

In many microchannel electroplating processes, the final enclosure of the channels is achieved by overgrowing or "mushrooming" the gold on top of the resist until channel walls meet ${ }^{9}$ or by fabricating the top and bottom pieces separately and spot welding them together. The mushrooming technique is simple and fast, but it results in plating an extra thick top layer of at least half the channel thickness. In addition, this technique limits the achievable design geometries preventing optimal performance of the device. In contrast, the bonding technique allows for control over device height and arbitrary device geometry. However, it is a very slow and expensive process to isolate, align, and weld each individual device, and can result in air bubbles often remaining trapped between layers, creating nonbonded spots, which create leakages in the final device.

In order to avoid these limitations, we developed a process to seal the channels using a thin gold seed layer deposited via evaporation prior to electroplating of the third structural layers [Fig. 2(d)]. This provides a more evenly distributed current path in the electroplating bath, yielding more control of the final thickness of the cover layer compared to overgrowing the channels until they seal, and eliminates the "mushroom" topography usually associated with using such techniques.

After the third layer (cover) fabrication, all the photoresist in the device is eliminated in an ultrasonic acetone bath with mild agitation for $12 \mathrm{~h}$, and the device is released from the handle wafer with a chrome etchant [Fig. 2(e)]. No visible photoresist residue or chrome was observed in the final device, although further inspection of the gold material (elemental analysis) is necessary.

To investigate the fabricated microchannels, the silicon wafer was diced across the channels. Figure 3 shows an image of the fabricated microchannel cross section with gold sealing. The aspect ratio depends solely on the thick- 


\section{$\mathrm{JM}^{3}$ LETTERS}

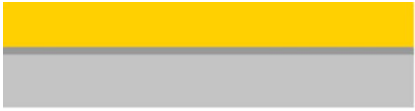

(a)

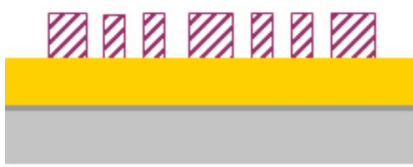

(b)

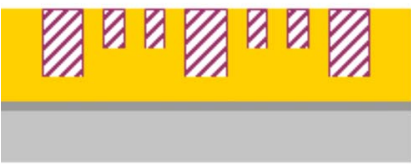

(c)

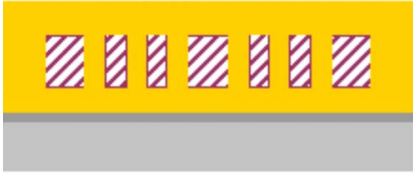

(d)

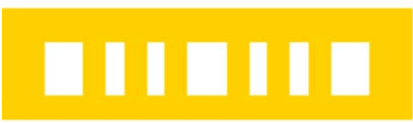

(e)
Gold

Adhesion Layers

Silicon Wafer

Photoresist

Fig. 2 Shunt fabrication process: (a) evaporation of adhesion and seed layers followed by thick resist lithography and electroplating of base layer; (b) thick resist molding for channels (layer 2); (c) electroplating of layer 2; (d) evaporation of gold seed layer, thick resist patterning, and electroplating of the top layer; and (e) removal of photoresist, etching of chrome adhesion layer to release devices.

ness of the sacrificial material. As explained before, deeper channels will have more stringent process parameters, but the fabrication steps will remain the same. The release time of the device simply depends on the thickness of the chromium adhesion layer as well as the width of the device. However, this release process is several days long. Prior to the release, the mild ultrasonic agitation used to etch away the photoresist lasts $15 \mathrm{~min}$.

\section{Conclusion}

We have successfully demonstrated a cost-effective, biocompatible, microchannel fabrication technique capable of producing a variety of metal devices. The width, depth, and length can be precisely controlled by the combination of oxygen plasma ashing, standard photolithography, and electroplating. The fabrication method described here is scalable (i.e., several more layers could be added to the device). However, the lithography process step for each layer will be different and needs to be carefully investigated. The technique of applying a seed layer on top the channels prior to the sealing improves the roughness of the top layers, improves electroplating rates, and enables arbitrary design geometries. The proposed process is suitable for large volume production and is scalable.

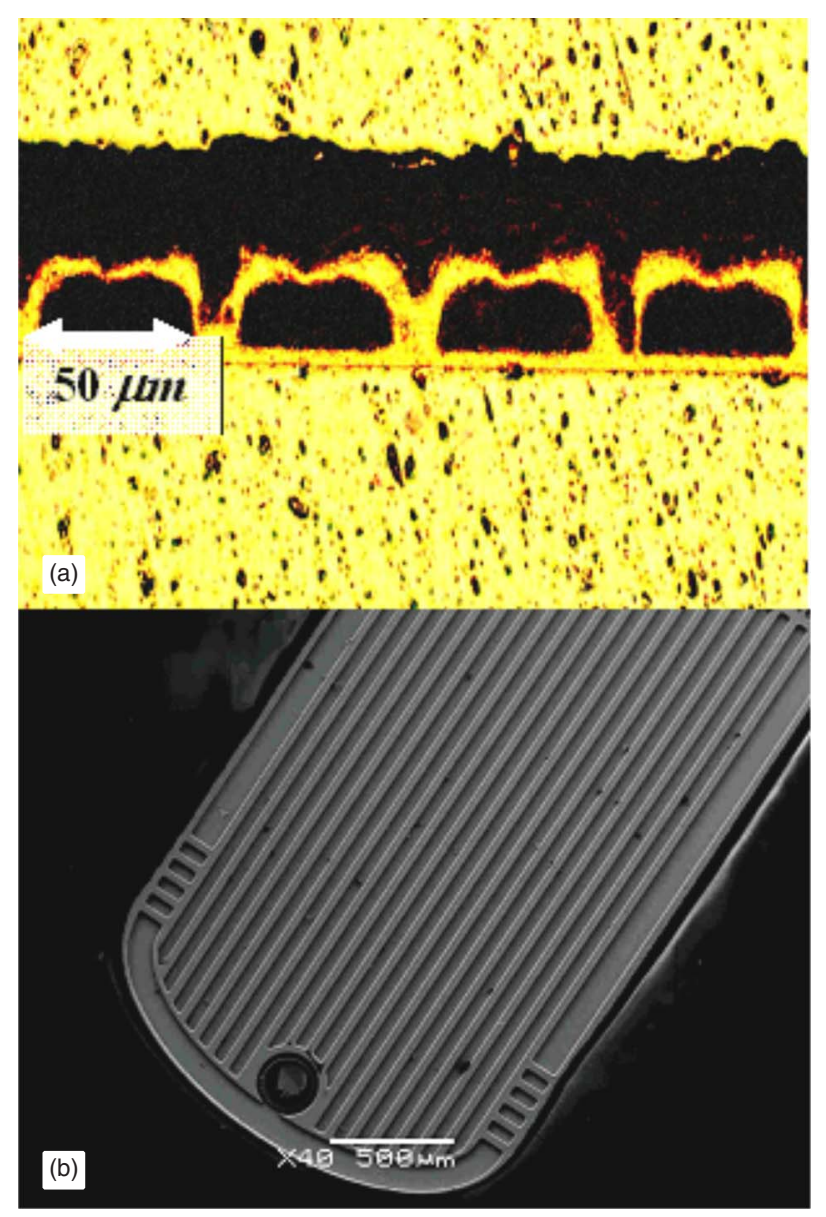

Fig. 3 Microchannels: (a) cross section of microchannels, and (b) scanning electron microscope image of layers 2 (channels) and 1 (base).

\section{Acknowledgments}

The authors would like to acknowledge the partial support from SOIX, Inc., for this project.

\section{References}

1. C. Tello, "The SOLX micro-shunt: IOP reduction without a bleb," Ophthalmol. Man. (Apr. 2006)

2. R. W. Tjerkstra, M. J. de Boer, J. W. Berenschot, J. G. Gardeniers, M C. Elwenspoek, and A. van den Berg, "Etching technology for microchannels," in Proc. IEEE MEMS Workshop, Nagoya, Japan (1997).

3. R. T. Howe, "Surface micromachining for micro sensors and micro actuators," J. Vac. Sci. Technol. B 6, 1808-1813 (1988).

4. K. B. Lee and L. Lin, "Surface micromachined glass and polysilicon microchannels using MUMPs for BioMEMS applications," Sens. Actuators, A 111, 44-50 (2004).

5. M. J. de Boer, R. W. Tjerkstra, J. W. Berenschot, H. V. Jansen, G. J. Burger, J. G. Gardeniers, M. C. Elwenspoek, and A. van den Berg, "Micromachining of buried micro channels in silicon," J. Microelectromech. Syst 9(1), 94-103 (2000).

6. S. Tuomikoski and S. Franssila, "Free-standing SU-8 microfluidic chips by adhesive bonding and release etching," Sens. Actuators, A 120(2), 408-415 (2005)

7. I. Papautsky, J. Brazzle, H. Swerdloe, and B. Frasier, "A lowtemperature IC-compatible process for fabricating surfacemicromachined metallic microchannels," J. Microelectromech. Syst 7(2), 267-272 (1998).

8. F. M. White, Fluid Mechanics, 4th ed., New York (1999).

9. Y. Joo, K. Dieu, and C.-J. Kim, "Fabrication of monolithic microchannels for IC chip cooling," in Proc. IEEE 6, 362-367 (1995). 